\title{
Direct constraints on diffusion models from cosmic-ray positron data: Excluding the Minimal model for dark matter searches
}

\author{
Julien Lavalle \\ Laboratoire Univers \& Particules de Montpellier (LUPM), \\ CNRS-IN2P3 \& Université Montpellier II (UMR-5299), \\ Place Eugène Bataillon, F-34095 Montpellier Cedex 05 — France* \\ David Maurin \\ LPSC, Université Grenoble-Alpes, CNRS/IN2P3, \\ 53 avenue des Martyrs, F-38026 Grenoble - France \\ Antje Putze \\ LAPTh, Université de Savoie, CNRS, 9 chemin de Bellevue B.P.110, F-74941 Annecy-le-Vieux - France
}

\begin{abstract}
Galactic Cosmic-ray (CR) transport parameters are usually constrained by the boron-to-carbon ratio. This procedure is generically plagued with degeneracies between the diffusion coefficient and the vertical extent of the Galactic magnetic halo. The latter is of paramount importance for indirect dark matter (DM) searches, because it fixes the amount of DM annihilation or decay that contributes to the local antimatter CR flux. These degeneracies could be broken by using secondary radioactive species, but the current data still have large error bars, and this method is extremely sensitive to the very local interstellar medium properties. Here, we propose to use the low-energy $\mathrm{CR}$ positrons in the $\mathrm{GeV}$ range as another direct constraint on diffusion models. We show that the PAMELA data disfavor small diffusion halo $(L \lesssim 3 \mathrm{kpc})$ and large diffusion slope models, and exclude the minimal ( $\mathrm{min}$ ) configuration (Maurin et al. 2001, Donato et al. 2004) widely used in the literature to bracket the uncertainties in the DM signal predictions. This is complementary to indirect constraints (diffuse radio and gamma-ray emissions) and has strong impact on DM searches. Indeed, this makes the antiproton constraints more robust while enhancing the discovery/exclusion potential of current and future experiments, like AMS-02 and GAPS, especially in the antiproton and antideuteron channels.
\end{abstract}

\section{INTRODUCTION}

The theoretical understanding of cosmic-ray (CR) transport relies on diffusion of charged particles off magnetic turbulences and has been established for decades [1-3]. In this picture, CRs are confined in an extended region that encompasses the Galactic disk, which can be assumed as a homogeneous magnetic cylinder at first order. Therein the diffusion tensor is reduced to a rigiditydependent scalar (homogeneous and isotropic diffusion). Yet, it is only very recently that we have been able to start probing the fine structure of CR phenomenology. With the advent of space experiments like PAMELA [49], Fermi [10, 11], and more recently AMS-02 [12], the physics of Galactic CRs has just entered the precision era. CR measurements also provide very interesting probes of exotic physics. In particular, the survey of antimatter CR species may unveil traces of dark matter (DM) annihilation or decay in the Galaxy (e.g., Refs. [13-15]).

The background to DM searches mostly comes from secondary CRs, i.e. those CRs produced from nuclear interactions between the CR nuclei and the interstellar gas. This secondary component is used to constrain the CR transport model parameters, as the ratio of secondary-toprimary CR nuclei depends very little on the properties of the primaries at their sources, while very strongly on the transport history $[16,17]$. The most widely used ratio is $\mathrm{B} / \mathrm{C}$ [18-25], although other ratios like ${ }^{2} \mathrm{He} /{ }^{1} \mathrm{H}$ and ${ }^{3} \mathrm{He} /{ }^{4} \mathrm{He}$ are equally powerful [26]. Once the transport parameters are set (from $\mathrm{B} / \mathrm{C}$ analysis), one can fully predict the fluxes of the other secondary species (same propagation history) provided the relevant production cross sections are known. Such calculations have been done for secondary positrons [27-31], antiprotons [32-35], and antideuterons [36]. For all but the positron case, for which energy losses play a major role in contrast to nuclei, these computations are poorly sensitive to the theoretical uncertainties affecting the transport parameters in spite of the large degeneracies induced by the B/C analysis $[19,20]$.

In 2-zone diffusion models, a large uncertainty for DM searches stems from the degeneracy between the normalization of the diffusion coefficient ${ }^{1} K_{0}$ and half the vertical extent of the diffusion halo $L$. Indeed, the $\mathrm{B} / \mathrm{C}$ data mostly constrain the $\mathrm{CR}$ escape time $\propto L / K_{0}$ - as all other secondary-to-primary ratios of stable nuclei origi-

\footnotetext{
${ }^{1}$ We assume $K(\mathcal{R} \equiv|p / q|)=\beta K_{0}(\mathcal{R} / 1 \mathrm{GV})^{\delta}$ in the following - where $p$ is the momentum, $\beta$ the velocity, and $q$ the electric charge.
}

\footnotetext{
*lavalle@in2p3.fr

$\dagger$ dmaurin@lpsc.in2p3.fr

$\ddagger$ putze@lapth.cnrs.fr
} 
nating from the Galactic disk. In contrast, DM-induced CRs are produced all over the diffusion halo (and outside), and its size $L$ has a strong impact on the signal predictions: their flux roughly scales like $\sim L^{2} / K_{0}$ (assuming a constant DM density, a fairly good approximation for qualitative understanding). This picture is valid whenever the transport is dominated by spatial diffusion.

In Ref. [37], the Authors proposed two extreme configurations to bracket the theoretical uncertainties on the DM signal predictions, dubbed min and max, relying on the $\mathrm{B} / \mathrm{C}$ analysis performed in [20]. The former (latter) is featured by a very small (large) diffusion zone with $L=1$ (15) kpc, and is associated with low (large) signal predictions. In practice, the min model is usually invoked to minimize the antiproton constraints on DM candidates, while $\max$ is used to promote detectability - the relative difference between the two almost reaches two orders of magnitude in terms of flux predictions. Such a large range for $L$ strongly affects the antimatter CRs as reliable probes of the DM parameter space. This is particularly important in the light-intermediate WIMP mass range (10-100 GeV), where antiprotons could be used to place severe constraints on WIMPs annihilating or decaying into quarks [38].

There are serious hints that $L$ should be larger than $\sim 1$ kpc, but no stringent bounds so far. Radioactive species are insensitive to $L$ at low energy because their lifetime is shorter than the diffusion time to reach the halo boundary. Using for instance ${ }^{10} \mathrm{Be} /{ }^{9} \mathrm{Be}$ breaks the $K_{0} / L$ degeneracy and sets constraints on $L[19,21]$. However, it was shown in several studies that this method is very sensitive to the modeling of the local interstellar medium (ISM), and strongly affected by the presence of a local underdensity, known as the local bubble [39, 40]: this relaxes the lower bound on $L$, depending on the size of the underdense region [24, 41]. There are also other, while more indirect, hints for larger values for $L$, coming e.g. from calculations of the diffuse Galactic gamma-ray [42] or radio emissions [43-45]. Nevertheless, predictions of these observables rely on more ingredients (line-of-sight integrals depending on the astrophysical source, ISM, and/or magnetic field distributions).

In this paper, we propose to use the low-energy secondary CR positrons as an additional direct constraint on $L$. We exploit the fact that the propagation history of positrons is different from that of nuclei, due to energy losses. This typically shortens the mean free path of positrons, and the dependence on $L$ is milder. In particular, the secondary positron flux roughly scales like $\sim 1 / \sqrt{K_{0}}$, allowing us to place a lower bound on $L$ from the current positron data, assuming the B/C-induced relation between $K_{0}$ and $L$. We will only rely on secondary positrons, though it is clear that a primary component is also expected from recent measurements of the positron fraction [4, 10, 12]. Our approach, suggested in [46], is complementary to the study carried out later in Ref. [45], though with a different propagation treatment, for which the main constraints came from the diffuse radio emission data. We first briefly discuss the propagation modeling and relevant parameters, then sketch our statistical analysis method before going to the results and conclusion.

\section{TRANSPORT OF COSMIC-RAY POSITRONS}

We wish to constrain small 2D diffusion halo models, with $L \sim 1 \mathrm{kpc}$. In this context, as observers located at $8 \mathrm{kpc}$ from the Galactic center while far enough from the radial border located at $\sim 10-15 \mathrm{kpc}$ from us, we can neglect the radial escape. We then restrict ourselves to a much simpler 1D problem along the vertical axis. Secondary positrons originate in the Galactic disk from inelastic scattering processes of primary CRs off the interstellar gas. This source term is well constrained inside a radius $\sim 1 \mathrm{kpc}$ around the Earth since the CR flux barely varies over such a distance, and the average gas density confined in the disk is well estimated. It can be approximated to $\mathcal{Q}(E, \vec{x})=2 h n_{\text {ism }} \delta(z) Q_{0}(E)$, where $n_{\text {ism }}=1 \mathrm{~cm}^{-3}$ is the ISM gas density, and $h=100 \mathrm{pc}$ is half the disk width. The energy dependence is carried by $Q_{0}(E)=$ $4 \pi \sum_{i, j} f_{j} \int d T\left(d \phi_{\mathrm{cr}, \mathrm{i}}(T) / d T\right)\left(d \sigma_{i j \rightarrow e^{+}}(E) / d E\right)$, which convolves the CR flux (species $i$ ) with the ISM gas (species $j$, featuring a fraction $f_{j}$ ).

In the following, we will stick to the formalism presented in [28-30] for the calculation of secondary positrons. In [28] (see their Fig. 10), it was shown that transport configurations implying both reacceleration and convection resulted in a prominent low-energy bump around $1 \mathrm{GeV}$ in the secondary positron flux. To be conservative in our comparisons with the data, we consider that positrons are only driven by spatial diffusion and energy losses. This actually significantly reduces the computational time. We can define an energy-dependent propagation scale $\lambda$ as:

$$
\begin{aligned}
\lambda^{2}\left(E, E_{s}\right) & =4 \int_{E}^{E_{s}} d E^{\prime} \frac{K\left(E^{\prime}\right)}{b\left(E^{\prime}\right)} \\
& =(3.56 \mathrm{kpc})^{2} \frac{K_{0}}{10^{-2} \mathrm{kpc}^{2} / \mathrm{Myr}} \frac{\tau_{l}}{10^{16 \mathrm{~s}}} \\
& \times\left\{\frac{\left(E / E_{0}\right)^{(\delta-\alpha+1)}}{\alpha-\delta-1}\left[1-\left(E / E_{s}\right)^{\alpha-\delta-1}\right]\right\}
\end{aligned}
$$

where $E\left(E_{s} \geq E\right)$ is the observed (injected) positron energy, and where we have used $b(E)=-d E / d t=$ $\left(E_{0} / \tau_{l}\right)\left(E / E_{0}\right)^{\alpha}$ for the energy losses. For GeV positrons, losses are dominated by inverse Compton and synchrotron processes and the Thomson approximation holds, such that $\alpha=2$ and $\tau_{l} \simeq 10^{16} \mathrm{~s}\left(\right.$ for $\left.E_{0}=1 \mathrm{GeV}\right)$ to a very good approximation (we will use a more accurate form for $b(E)$ in practice). The propagation scale $\lambda$ allows us to write the Green function for the positron transport:

$$
\mathcal{G}_{1 \mathrm{D}}\left(E, z \leftarrow E_{s}, z_{s}\right)=\frac{e^{-\left|\frac{z-z_{s}}{\lambda}\right|^{2}}}{b(E) \sqrt{\pi \lambda^{2}}} \stackrel{z, z_{s} \rightarrow 0}{\longrightarrow} \frac{1}{b(E) \sqrt{\pi \lambda^{2}}}(3)
$$


For small- $L$ models, boundary effects are important. When both the observer and the source are confined into the disk $\left(z, z_{s}=0\right)$, the following series expansions can be used in the regime $\lambda \gtrsim L$, relevant at low energy [47]:

$$
\begin{aligned}
\mathcal{G}_{1 \mathrm{D}, \mathrm{Helm}}\left(E \leftarrow E_{s}\right) & =\frac{1}{b(E) L} \sum_{n=1}^{n=+\infty} e^{-\left|\frac{(2 n-1) \pi \lambda}{4 L}\right|^{2}} \\
& \approx \frac{e^{-\left|\frac{\pi \lambda}{4 L}\right|^{2}}+e^{-\left|\frac{3 \pi \lambda}{4 L}\right|^{2}}}{b(E) L}
\end{aligned}
$$

If we express the $\mathrm{B} / \mathrm{C}$ correlation between $K_{0}$ and $L$ as $K_{0}=\kappa L$, with $\kappa \sim 10^{-3} \mathrm{kpc} / \mathrm{Myr}$ [20], we can read off the dependence of the $\mathrm{CR}$ positron density on $L$ from the leading term:

$$
\mathcal{G}_{1 \mathrm{D}, \mathrm{Helm}}\left(E \leftarrow E_{s}\right) \approx \frac{e^{-\frac{\kappa \tau_{l} f\left(E, E_{s}\right)}{L}}}{b(E) L},
$$

where $f\left(E, E_{s}\right) \approx\left(E / E_{0}\right)^{(\delta-1)} /(1-\delta) \approx 2 / \sqrt{E /(\mathrm{GeV})}$. Interestingly, the pre-factor is proportional to $1 /(b(E) L)$, where it appears that a small $L$ may lead to a large secondary positron flux.

The additional impact of $\delta$ can be understood from the spectral shape predicted for the secondary positron flux [29], which roughly scales as $E^{-\tilde{\gamma}}$, with the spectral index $\tilde{\gamma} \simeq \gamma+(1+\delta) / 2$, where $\gamma \approx 2.7$ is the source index associated with secondary positron production. Therefore, the smaller $\delta$ the harder the secondary positron spectrum.

Finally, we note that since we focus on the GeV energy range, our results are sensitive to solar modulation effects that we will include in the force-field approximation [48].

\section{STATISTICAL ANALYSIS}

Given the flux data $\phi_{\text {data }}(E)$ and associated statistical error $\sigma_{\phi_{\text {data }}}(E)$, we wish to constrain only those transport models leading to secondary positron fluxes in excess with respect to the data. For each data point at kinetic energy $E_{i}$, the number of standard deviations in a one-sided hypothesis test of a Gaussian variate, i.e. the $Z$-score, is calculated:

$$
Z_{i}=\frac{\phi_{\text {model }}\left(E_{i}\right)-\phi_{\text {data }}\left(E_{i}\right)}{\sigma_{\phi_{\text {data }}}\left(E_{i}\right)}
$$

where $\phi_{\text {model }}\left(E_{i}\right)$ is the modulated flux estimated for a given parameter set $\left\{K_{0}, L, \delta\right\}$. In a subsequent step, the individual p-value $p_{i}$ is estimated only for data points with a positive $Z_{i}$ :

$$
p_{i}=1-\Phi\left(Z_{i}\right)=\frac{1-\operatorname{erf}\left(Z_{i} / \sqrt{2}\right)}{2}
$$

where $\Phi(Z)$ is the cumulative distribution function of the Gaussian distribution. The independent $p_{i}$ values of a given model are eventually combined into a single test statistic $X$ using Fisher's method [49]:

$$
X=-2 \sum_{i}^{n} \log p_{i}
$$

$X$ follows a $\chi_{2 n}^{2}$ with $2 n$ degrees of freedom from which a p-value for the global hypothesis can be obtained:

$$
p=1-\frac{\gamma\left(n, \chi_{2 n}^{2} / 2\right)}{\Gamma(n)},
$$

where $\gamma$ and $\Gamma$ are the lower incomplete and complete gamma functions, respectively.

In the following, we will use an exclusion criterion of $p<0.001345$, which corresponds to exceeding the data by $3-\sigma$ or more.

\section{RESULTS AND DISCUSSION}

The propagation framework is that defined in [28, 29], including the energy loss parameters. As we disregard reacceleration and convection, a transport model is defined by $K_{0}, \delta$, and $L$, which we vary in the ranges $K_{0} / L \in\left[10^{-3}, 10^{-2}\right] \mathrm{kpc} / \mathrm{Myr}, \delta \in[0.2-0.9]$, consistent with the $\mathrm{B} / \mathrm{C}$ constraints [20].

To cover the great variety of possible diffusion model parameters, 500000 parameter sets have been uniformly drawn in the ranges defined above. We have used the PAMELA data [9] associated with a solar modulation potential of $520 \mathrm{MV}$ relevant to the data taking period [50]. We also checked our method with the unpublished AMS02 data presented at ICRC 2013, but will only comment on the trend waiting for the published results. A $2 \mathrm{D}$ projection in the $\log \left(K_{0} / L\right)-L$ plane of (i) the models parameters drawn, (ii) those allowed by the PAMELA data $\left(\forall i: Z_{i} \leq 0\right)$, and (iii) those leading to excess with respect to one or more data points $\left(\exists i: Z_{i}>0\right)$, are shown in the left, middle, and right panels of Fig. 1, respectively - the color code indicates the density of propagation models per $2 \mathrm{D}$ bin. The AMS-02 data from ICRC 2013 would lead to similar trends. It is clear that the positron data constrain extensively low values of $L$ and $\log \left(K_{0} / L\right)$ independent of the spectral index $\delta$ (white area in the middle plot).

The influence of $\delta$ can be understood from Fig. 2. In the left (middle) panel, we show the predicted fluxes giving $Z>0$ while not excluded (excluded) by our analysis, for different slices in $\delta$ (the $Z<0$ models would give fluxes that spread over a large area below the data points). We see that the larger $\delta$, the more constraining the low-energy data points, as it can be expected from the spectral dependence in $\delta$ (see Sec. II). In contrast, predictions from a more gradual slope of $\delta \sim 0.3$ follow the data more closely (in the $\mathrm{GeV}$ range) and are hence more equally constrained by the PAMELA data points 1 to 14 (i.e. 1.64 to $33.1 \mathrm{GeV}$, out of a total of 17 data 

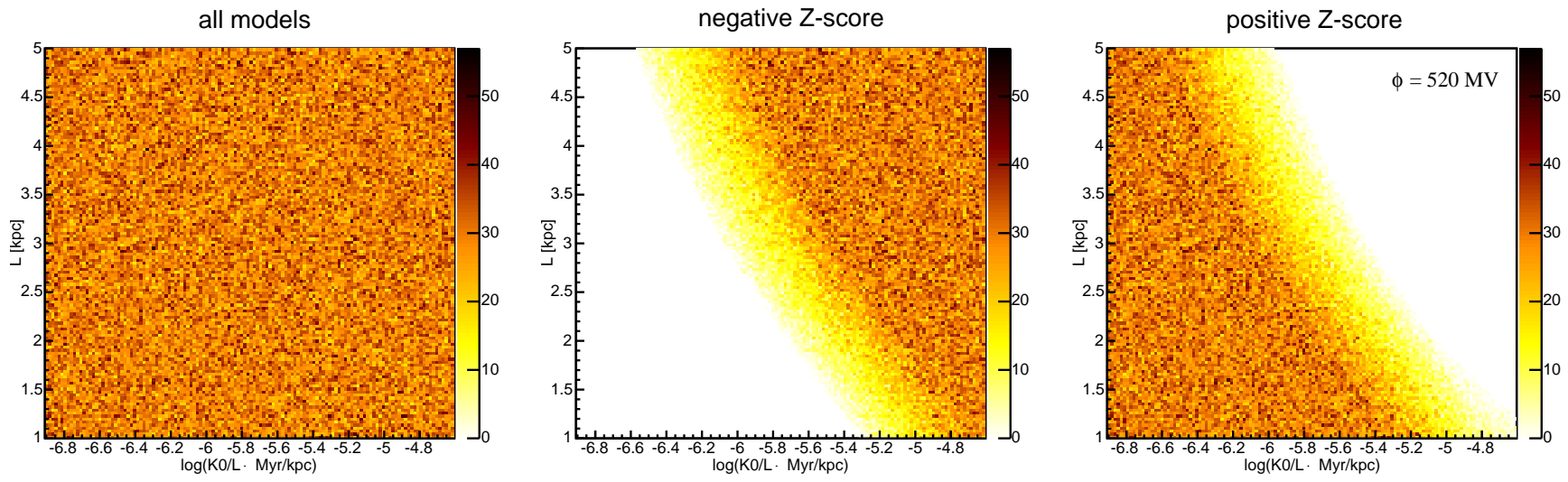

FIG. 1: 2D histograms for $L$ vs $\log \left(K_{0} / L\right)$ for $\delta \in[0.2-0.9]$. The color code indicates the density of propagation models per $2 \mathrm{D}$ bin.
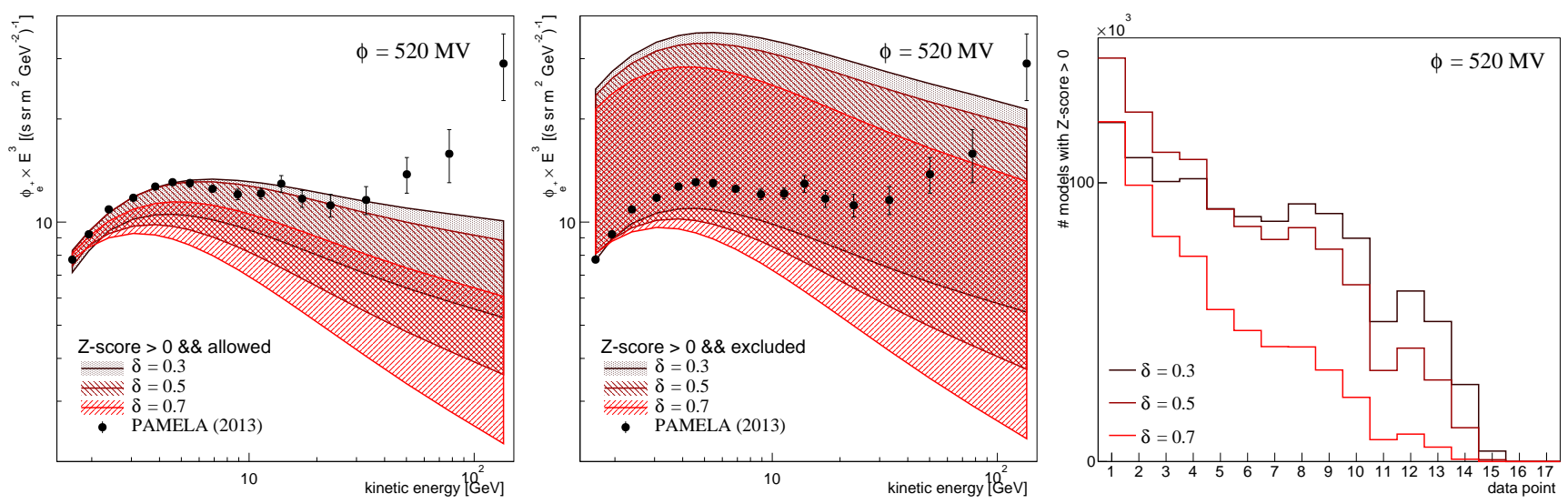

FIG. 2: Left: contours of the secondary positron flux predictions in excess with one or more data points, still allowed by our analysis. Middle: the same for models excluded by our analysis. Right: histogram of models with $Z_{i}>0$ excluded by our analysis for a given PAMELA data point (17 data points from 1.64 to $135 \mathrm{GeV}$ ).

\begin{tabular}{cccccc}
\hline & $\delta$ & $\begin{array}{c}K_{0} \\
\left(\mathrm{kpc}^{2} \mathrm{Myr}^{-1}\right)\end{array}$ & $\begin{array}{c}\mathrm{L} \\
(\mathrm{kpc})\end{array}$ & $\begin{array}{c}V_{c} \\
\left(\mathrm{~km} \mathrm{~s}^{-1}\right)\end{array}$ & $\begin{array}{c}V_{A} \\
\left(\mathrm{~km} \mathrm{~s}^{-1}\right)\end{array}$ \\
\hline max & 0.46 & 0.0765 & 15 & 5 & 117.6 \\
med & 0.70 & 0.0112 & 4 & 12 & 52.9 \\
min & 0.85 & 0.0016 & 1 & 13.5 & 22.4 \\
\hline
\end{tabular}

TABLE I: Transport parameters associated with the max, med and min DM-induced $\bar{p}$ and $\bar{d}$ fluxes.

points up to $135 \mathrm{GeV})$. In the right panel, we display the data points with $Z_{i}>0$ for the models excluded from our nominal analysis, for different slices in $\delta$. The trend explained above is explicit in this plot.

In Fig. 3, we show the $3-\sigma$ exclusion curves obtained in the $\log \left(K_{0} / L\right)-L$ plane, i.e. our primary result. Plain lines are exclusion curves for different values of $\delta$. Dashed lines show how the result changes if the secondary flux predictions are increased by a global factor of $30 \%$, a rough way to account for a possible primary positron component at low energy. In the left panel, we adopt the nominal value of $520 \mathrm{MV}$ for the solar modulation.
In the right panel, we take a very conservative viewpoint to secure the results against systematic effects possibly coming from the solar modulation modeling or other lowenergy effects, (i) by considering only the data above $\sim 2$ $\mathrm{GeV}$, and (ii) by overstating the solar modulation potential up to $700 \mathrm{MV}$.

We report on the same plots the B/C constraints obtained in Ref. [20], in the form of bands corresponding to different slices in $\delta$. There are several reasons as for why most recent studies are not used. First, although powerful statistical tools have been employed since [24-26] to sample the most probable regions of the parameter space, we take a wider and more conservative range such as that given in Ref. [20]. This allows us to overcome possible systematic uncertainties arising in the determination of the transport parameters [24]. Second, benchmark models min, med, and max of Ref. [37] (see Table I), widely used in the DM literature, are based on the parameters found in [20]. The min and med models are shown in both panels of Fig. 3, while the max model falls outside with $L=15 \mathrm{kpc}$. They belong to different B/C bands. 

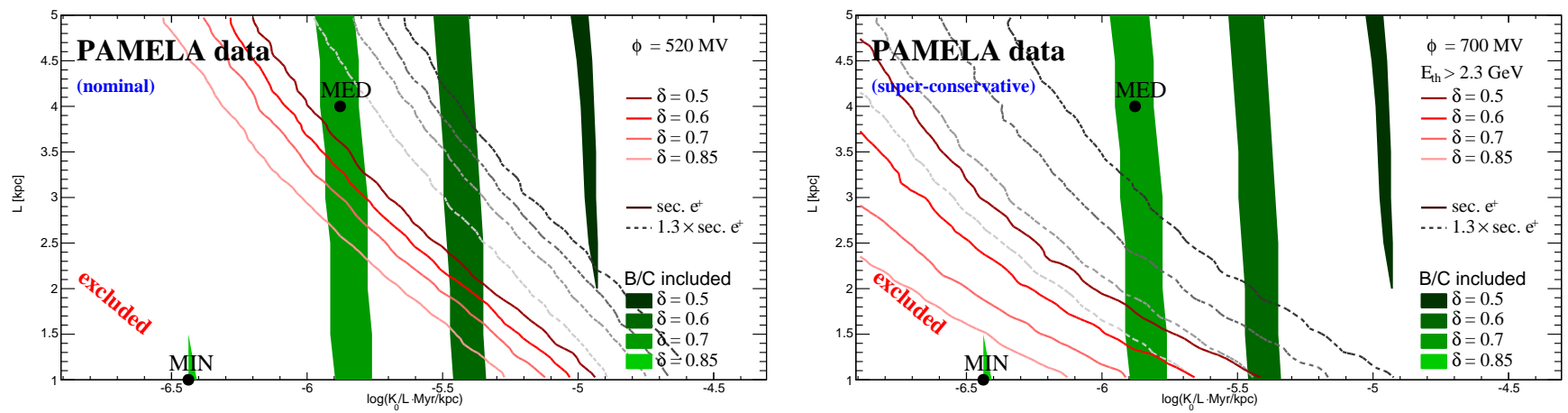

FIG. 3: Constraints on propagation parameters in the $\log \left(K_{0} / L\right.$ in $\left.[\mathrm{kpc} / \mathrm{Myr}]\right)-L[\mathrm{kpc}]$ plane. Lines are constraints from the positron flux (downward regions are excluded). Green filled contours are allowed by B/C data. The min and med models of Ref. [37] (see Tab. I) are indicated by a filled black circle. Left panel: positron contours (from PAMELA data) for a realistic modulation level of $\phi=520 \mathrm{MV}$. Dashed lines correspond to the limits if the secondary positron prediction is increased by $30 \%$ to mimic a primary component. Right panel (super-conservative): Same but with a solar modulation of $700 \mathrm{MV}$ and considering only data points $\gtrsim 2 \mathrm{GeV}$.

An important feature of Fig. 3 is that the positron and $\mathrm{B} / \mathrm{C}$ constraints are almost orthogonal in the $\log \left(K_{0} / L\right)$ $L$ plane, and thereby uncorrelated. This is a strength of this approach and it can complement the one relying on radioactive species, which has completely different systematics. As we can see in the left panel (nominal result), the min model is completely excluded. This is important because this model is very often used to bracket the propagation uncertainties in indirect DM searches. More generally, models with large values of $\delta(\sim 0.8)$, generically associated with small diffusion halos $(L \sim 1 \mathrm{kpc})$, are excluded by our analysis. Smaller values of $\delta$ are featured by larger $K_{0} / L$ ratios. For example, we see that for the $\mathrm{B} / \mathrm{C}$ band containing the med model $(\delta \sim 0.7)$, the positron constraint reads $L \gtrsim 3 \mathrm{kpc}$, while a less severe $L \gtrsim 2.3 \mathrm{kpc}$ is obtained for smaller diffusion slopes. The dashed lines indicate how these limits would move if a primary positron component contributed an additional $30 \%$ to the secondary flux at low energy ${ }^{2}$, leading to much larger lower bounds in $L$. Nevertheless, though primary positrons are present in the $\sim 10-100 \mathrm{GeV}$ data, their origin remains unclear, and their contribution at low energy can hardly be predicted. Still, confronting the secondary positron predictions to the data provides direct constraints on $L$ that rely on fewer assumptions (e.g. on the Galactic magnetic or interstellar radiation fields) than, for example, those set from the diffuse radio or gamma-ray emissions, involving mostly primary electrons and positrons.

We have followed a conservative line by neglecting diffusive reacceleration, which would increase our predic-

\footnotetext{
2 This $30 \%$ is the approximate increase needed to saturate the positron data in propagation models with $L \sim 4 \mathrm{kpc}$, i.e. similar to the med configuration; spectral effects are neglected as the energy range relevant to get the limit is narrow.
}

tions around 1.5-2 GeV as $\delta$ decreases [28]; we have also neglected any primary component. As we deal with lowenergy positrons, the solar modulation modeling and intensity may also have significant impact on the limit derived. Although rather simplistic, the force-field approximation is expected to be accurate within $\sim 10-20 \%$ above $100 \mathrm{MeV}$ [51]. In our nominal analysis, we took a modulation potential of $520 \mathrm{MV}$, and used all PAMELA data points down to $1.64 \mathrm{GeV}$ - the lowest energy data points strongly constrain large- $\delta$ models. To check the robustness of our results, we adopt the radical option (i) to increase the modulation potential up to $700 \mathrm{MeV}$ and (ii) to remove the first data points from the analysis, setting a threshold at $\sim 2 \mathrm{GeV}$ (more precisely $2.38 \mathrm{GeV}$, $3^{\text {rd }}$ PAMELA data point). Such an option is extreme, so the corresponding results are to be taken as superconservative. They are displayed in the right panel of Fig. 3. The limits on $L$ are obviously weaker, but the min model remains excluded, as propagation models with large values of $\delta(\sim 0.8)$.

Similar limits on $L$ were obtained in other studies, e.g. Refs. [25] and [45]. In the former, the Authors mostly use the $\mathrm{B} / \mathrm{C}$ and ${ }^{10} \mathrm{Be} /{ }^{9} \mathrm{Be}$ ratios to constrain the $\mathrm{CR}$ transport parameters, and obtain the bound $L>3.2 \mathrm{kpc}(95 \%$ CL) from a Bayesian analysis. While consistent with our result, this work, relying on different observables, is based on several assumptions that might induce biases: a lowenergy spectral break in the primary source spectra and the absence of convection. Moreover, the presence of the local gas underdensity (local bubble) affects particularly this kind of studies [24, 41]. Our result has therefore his own strength as it is affected by different systematics, and may be viewed as slightly more conservative in the propagation setting. In Ref. [45], the Authors focus on the electron-positron CRs (primaries and secondaries), and derive a qualitative bound of $L \gtrsim 2 \mathrm{kpc}$ from 16 
diffusion models (4 sets of assumptions, each taken with $L=1,2,4$, and $8 \mathrm{kpc})$. Although they briefly mention the secondary positron effect, their result relies on the analysis of the latitude profile of the $408 \mathrm{MHz}$ radio emission, for which they further have to assume a model for the Galactic magnetic field. Again, our result is complementary: our propagation setup is more general, with different systematics, and our limit has an explicit statistical meaning.

\section{CONCLUSION}

In this paper, we have investigated the low-energy positron constraints $(\lesssim 10 \mathrm{GeV})$ on 2-zone CR propagation models. The obtained bounds are almost orthogonal to the $\mathrm{B} / \mathrm{C}$ constraints in the $L-\log \left(K_{0} / L\right)$ plane, which makes them particularly attractive. The primary result is that we exclude the min benchmark propagation model and more generally large diffusion indices $(\delta \gtrsim 0.8)$. We also strongly disfavor small diffusion halo models with $L \lesssim 3 \mathrm{kpc}$, the constraint weakening as the diffusion slope $\delta$ decreases. This has important consequences for DM studies that are often addressed in the frame of 2-zone models: this pushes the DM signal predictions toward larger values, which has a significant impact on the discovery/exclusion potentials of current and future experiments. This will be of particular interest for the searches in the antiproton and antideuteron channels with AMS02 and GAPS [52-54].

The strength of the proposed analysis, complementary to the $\mathrm{B} / \mathrm{C}$ or radioactive studies, will significantly improve when the AMS-02 data are released (more data points with smaller error bars). Preliminary calculations based on the preliminary AMS-02 data presented at the ICRC 2013 conference already give slightly stronger constraints on $L$. The next step of this work will be to implement a full study including all low-energy effects and combining the coming AMS-02 data on positrons and PAMELA and AMS-02 data on B/C.

We note that the PAMELA collaboration has just released its B/C data [55]. With an analysis based on a very limited number of parameters, the Authors constrain the diffusion slope $\delta$ to be in the range $\sim$ $[0.38-0.42]$. However, the choice of other free parameters (wind or no wind, low-energy dependence of the diffusion coefficient) has a strong impact on the result, and in particular on $\delta[24,56]$. It is important to perform a full scan of the parameter space with these new B/C data (awaiting the AMS-02 positron data and final $\mathrm{B} / \mathrm{C}$ analysis). Better constraints on $\delta$ should increase the discrimination power of positrons.

\section{ACKNOWLEDGMENTS}

We would like to thank L. Derome for useful discussions about statistical analysis methods. This work was partly funded by the French ANR, Programme BLANC DMAstro-LHC, Project ANR-12-BS05-0006, and by the Investissements d'avenir, Labex ENIGMASS.
[1] V. S. Berezinskii et al., Astrophysics of cosmic rays (Amsterdam: North-Holland, edited by Ginzburg, V.L., 1990).

[2] R. Schlickeiser, Cosmic ray astrophysics (Springer Berlin Heidelberg, 2002).

[3] A. W. Strong et al., Annual Review of Nuclear and Particle Science 57, 285 (2007), astro-ph/0701517.

[4] O. Adriani et al., Nature 458, 607 (2009), arXiv:0810.4995.

[5] O. Adriani et al., Phys. Rev. Lett. 105, 121101 (2010), arXiv:1007.0821 [astro-ph.HE].

[6] O. Adriani et al., Science 332, 69 (2011), arXiv:1103.4055 [astro-ph.HE].

[7] O. Adriani et al., Phys. Rev. Lett. 106, 201101 (2011), arXiv:1103.2880 [astro-ph.HE].

[8] O. Adriani et al., Astrophys. J. 770, 2 (2013), arXiv:1304.5420 [astro-ph.HE].

[9] O. Adriani et al., Phys. Rev. Lett. 111, 081102 (2013).

[10] A. A. Abdo et al., Phys. Rev. Lett. 102, 181101 (2009), arXiv:0905.0025.

[11] M. Ackermann et al., Phys. Rev. Lett. 108, 011103 (2012), arXiv:1109.0521 [astro-ph.HE].

[12] M. Aguilar et al., Phys. Rev. Lett. 110, 141102 (2013).

[13] J. Silk and M. Srednicki, Phys. Rev. Lett. 53, 624 (1984).

[14] T. A. Porter, R. P. Johnson, and P. W. Gra- ham, Ann.Rev.Astron.Astrophys. 49, 155 (2011), arXiv:1104.2836 [astro-ph.HE].

[15] J. Lavalle and P. Salati, Comptes Rendus Physique 13, 740 (2012), arXiv:1205.1004 [astro-ph.HE].

[16] D. Maurin, R. Taillet, and F. Donato, Astron.Astroph. 394, 1039 (2002), astro-ph/0206286.

[17] A. Putze, D. Maurin, and F. Donato, Astron.Astroph. 526, A101 (2011), arXiv:1011.0989 [astro-ph.GA].

[18] W. R. Webber, M. A. Lee, and M. Gupta, Astrophys. J. 390, 96 (1992).

[19] F. C. Jones, A. Lukasiak, V. Ptuskin, and W. Webber, Astrophys. J. 547, 264 (2001), astro-ph/0007293.

[20] D. Maurin, F. Donato, R. Taillet, and P. Salati, Astrophys. J. 555, 585 (2001), astro-ph/0101231.

[21] A. W. Strong and I. V. Moskalenko, Advances in Space Research 27, 717 (2001), astro-ph/0101068.

[22] A. M. Lionetto, A. Morselli, and V. Zdravkovic, JCAP 0509, 010 (2005), astro-ph/0502406.

[23] C. Evoli, D. Gaggero, D. Grasso, and L. Maccione, JCAP 10, 018 (2008), arXiv:0807.4730.

[24] A. Putze, L. Derome, and D. Maurin, Astron.Astroph. 516, A66 (2010), arXiv:1001.0551 [astro-ph.HE].

[25] R. Trotta, G. Johannesson, I. Moskalenko, T. Porter, R. R. de Austri, et al., Astrophys. J. 729, 106 (2011), arXiv:1011.0037 [astro-ph.HE]. 
[26] B. Coste, L. Derome, D. Maurin, and A. Putze, Astron.Astroph. 539, A88 (2012), arXiv:1108.4349 [astroph.GA].

[27] I. Moskalenko and A. Strong, Astrophys. J. 493, 694 (1998), astro-ph/9710124.

[28] T. Delahaye, R. Lineros, F. Donato, N. Fornengo, J. Lavalle, P. Salati, and R. Taillet, Astron.Astroph. 501, 821 (2009), arXiv:0809.5268.

[29] T. Delahaye, J. Lavalle, R. Lineros, F. Donato, and N. Fornengo, Astron.Astroph. 524, A51 (2010), arXiv:1002.1910 [astro-ph.HE].

[30] J. Lavalle, Mon.Not.Roy.Astron.Soc. 414, 985L (2011), arXiv:1011.3063 [astro-ph.HE].

[31] M. Di Mauro, F. Donato, N. Fornengo, R. Lineros, and A. Vittino, JCAP 4, 006 (2014), arXiv:1402.0321 [astroph.HE].

[32] F. Donato, D. Maurin, P. Salati, A. Barrau, G. Boudoul, and R. Taillet, Astrophys. J. 563, 172 (2001), astroph/0103150.

[33] T. Bringmann and P. Salati, Phys. Rev. D 75, 083006 (2007), astro-ph/0612514.

[34] F. Donato, D. Maurin, P. Brun, T. Delahaye, and P. Salati, Phys. Rev. Lett. 102, 071301 (2009), arXiv:0810.5292.

[35] G. Di Bernardo, C. Evoli, D. Gaggero, D. Grasso, and L. Maccione, Astroparticle Physics 34, 274 (2010), arXiv:0909.4548 [astro-ph.HE].

[36] F. Donato, N. Fornengo, and D. Maurin, Phys. Rev. D 78, 043506 (2008), arXiv:0803.2640.

[37] F. Donato, N. Fornengo, D. Maurin, P. Salati, and R. Taillet, Phys. Rev. D 69, 063501 (2004), astro$\mathrm{ph} / 0306207$.

[38] J. Lavalle, Phys. Rev. D 82, 081302 (2010), arXiv:1007.5253 [astro-ph.HE].

[39] R. Lallement, B. Y. Welsh, J. L. Vergely, F. Crifo, and D. Sfeir, Astron.Astroph. 411, 447 (2003).
[40] R. Lallement, J.-L. Vergely, B. Valette, L. Puspitarini, L. Eyer, and L. Casagrande, Astron.Astroph. 561, A91 (2014), arXiv:1309.6100 [astro-ph.GA].

[41] F. Donato, D. Maurin, and R. Taillet, Astron.Astroph. 381, 539 (2002), astro-ph/0108079.

[42] M. Ackermann et al., Astrophys. J. 750, 3 (2012).

[43] E. Orlando and A. Strong, Mon.Not.Roy.Astron.Soc. 436, 2127 (2013), arXiv:1309.2947 [astro-ph.GA].

[44] T. Bringmann, F. Donato, and R. A. Lineros, JCAP 1201, 049 (2012), arXiv:1106.4821 [astro-ph.GA].

[45] G. Di Bernardo, C. Evoli, D. Gaggero, D. Grasso, and L. Maccione, JCAP 3, 036 (2013), arXiv:1210.4546 [astro-ph.HE].

[46] J. Lavalle, J.Phys.Conf.Ser. 375, 012032 (2012), arXiv:1112.0678 [astro-ph.HE].

[47] J. Lavalle, J. Pochon, P. Salati, and R. Taillet, Astron.Astroph. 462, 827 (2007), astro-ph/0603796.

[48] L. J. Gleeson and W. I. Axford, Astrophys. J. 154, 1011 (1968).

[49] R. A. Fisher, Edinburgh: Oliver and Boyd, 1970, 14th ed. (1970).

[50] D. Maurin, A. Cheminet, L. Derome, A. Ghelfi, and G. Hubert, Adv.Space Res. in press (2014), 10.1016/j.asr.2014.06.021, arXiv:1403.1612 [astroph.EP].

[51] M. Potgieter, Living Reviews in Solar Physics 10, 3 (2013), arXiv:1306.4421 [physics.space-ph].

[52] C. J. Hailey et al., Advances in Space Research 51, 290 (2013).

[53] T. Aramaki et al., Astroparticle Physics 59, 12 (2014), arXiv:1401.8245 [astro-ph.HE].

[54] P. von Doetinchem et al., Astroparticle Physics 54, 93 (2014), arXiv:1307.3538 [astro-ph.IM].

[55] O. Adriani et al., ArXiv e-prints (2014), arXiv:1407.1657 [astro-ph.HE].

[56] D. Maurin, A. Putze, and L. Derome, Astron.Astroph. 516, A67 (2010), arXiv:1001.0553 [astro-ph.HE]. 